\title{
Putting Baby Safety Month in The Infant Safe Sleep Context
}

Barb Himes, IBCLC, CD

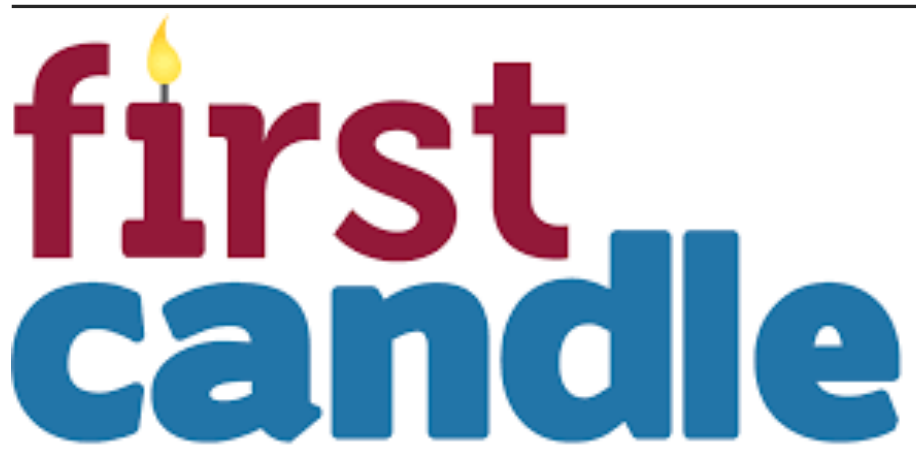

Saving babies. Supporting families.

First Candle's efforts to support families during their most difficult times and provide new answers to help other families avoid the tragedy of the loss of their baby are without parallel.

"September is Baby Safety Month, a reminder of the choices and challenges facing parents, extended families, and caregivers in creating both waking and sleeping safe environments for infants."

September is Baby Safety Month, a reminder of the choices and challenges facing parents, extended families, and caregivers in creating both waking and sleeping safe environments for infants. Sponsored annually by the Juvenile Product Manufacturers Association, Baby Safety Month is a time to focus on the prevention of accidents and the prevention of accidental suffocation and strangulation in bed (ASSB), a sleep-related infant death.

While the rate of Sudden Infant Death Syndrome (SIDS), another sleep-related infant death, has been falling, the number of babies dying from ASSB has risen from 4.38 per 100,000 live births in
1997 to 25.5 in 2019. From 1999 to 2015 alone, SIDS rates decreased $35.8 \%$, and ASSB rates increased $183.8 \%$, a sobering statistic. (1)

Accidental suffocation is now the most common cause of injury deaths for babies in the United States under one year old, with $82 \%$ being attributable to ASSB. The majority of these cases were due to soft bedding and occurred most often in an adult bed. (2)

This information underscores the need to address the causes of ASSB deaths - which are preventable -- and to counsel families on what to do when mother and baby leave the hospital.

\section{"Accidental suffocation is now the most} common cause of injury deaths for babies in the United States under one year old, with $82 \%$ being attributable to ASSB. The majority of these cases were due to soft bedding and occurred most often in an adult bed. (2)"

\section{Health Care Providers: A Critical Source}

Health care providers, particularly in NICUs, have an opportunity to educate families about safe sleep practices to undertake when they take their baby home. They are in a position to help new parents learn about the American Academy of Pediatrics' 2016 safe sleep guidelines and also to help them understand that there will be differences between what they may see practiced in the NICU and what should be done at home.

As reported in the July 2021 issue of Pediatrics, it is important for health care professionals to prepare families on how they can maintain their infant in a safe home sleep environment. (3) NICU infant needs may call for non-supine positioning, a practice that should be converted as soon as medically feasible (and well prior to hospital discharge) to sleep practices that are safe and appropriate for the home environment.

This includes compliance with the 2016 guidelines by placing in-

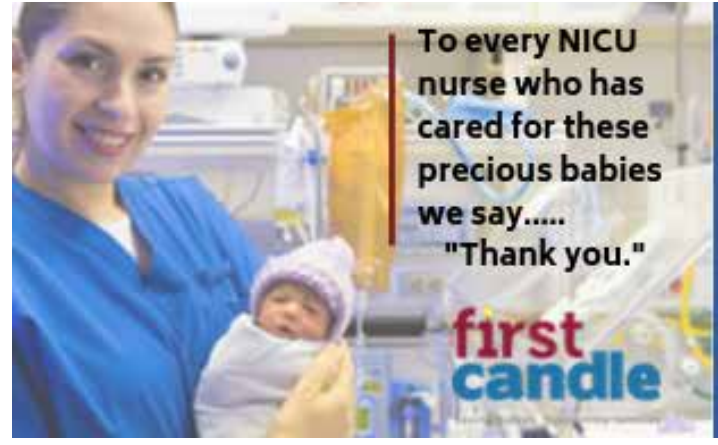

\section{Did you know that premature and low birth weight babies have a $4 \times$ greater risk for SIDS?}

At First Candle we're educating parents, grandparents and caregivers about safer sleep to make sure all babies reach their first birthday. Learn more at firstcandle.org 


\section{Trends in Sudden Unexpected Infant Death by Cause, 1990-2019}

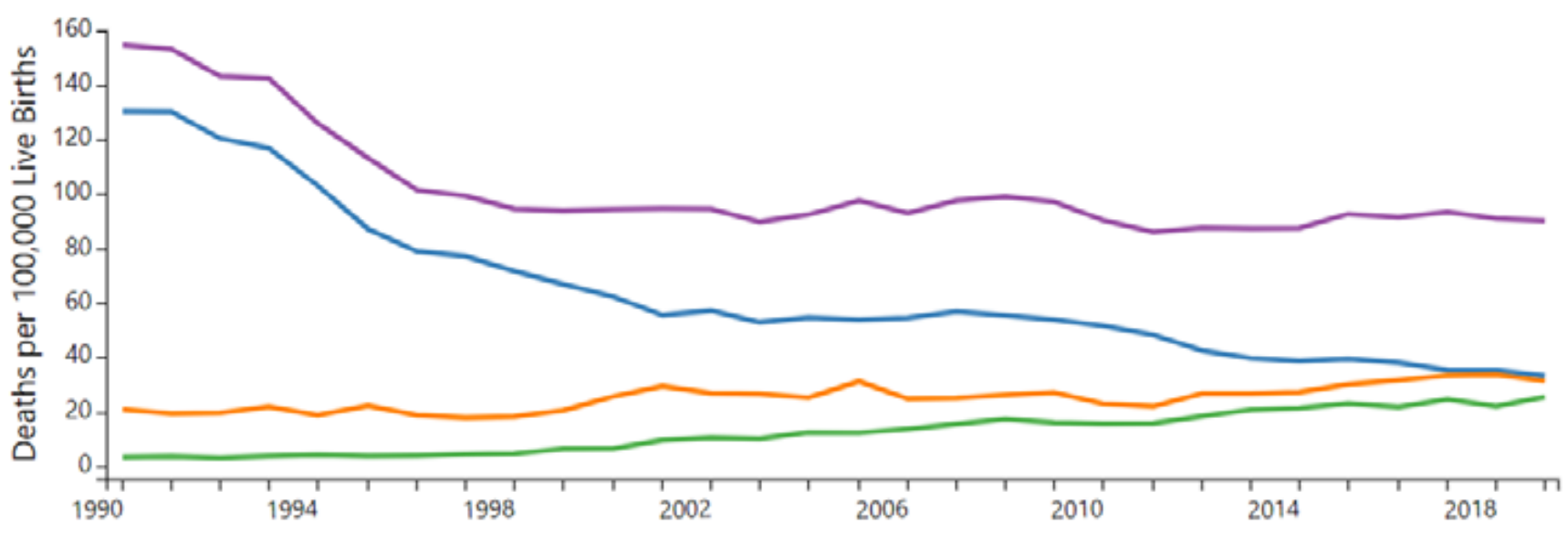

Year

Sudden infant death syndrome $\square$ Unknown cause $\square$ Accidental suffocation and strangulation in bed $\square$ Combined SUID rate

Source: https://www.cdc.gov/sids/data.htm

fants at home on a flat, firm sleep surface, such as a crib or bassinet, covered by a fitted sheet with no other bedding. Soft bedding and loose objects can cause airway obstruction and therefore increase the risk of rebreathing, SIDS, and suffocation. If bedding and positioners have been prescribed for developmentally sensitive care, they should nonetheless be removed from the sleep environment.

Safe sleep practices also extend to maternal and infant health through maternal adherence to keeping wellness visits and to gaining the benefits of breastfeeding and skin-to-skin contact. It is understood that safe sleep counsel will differ for non-NICU infants and NICU infants and that there may be individual considerations such as monitoring for Sudden Unexpected Postnatal Collapse (SUPC), depending upon maternal, infant, and birthing characteristics. (4)

New parents should be learning about infant safe sleep practices and concepts at multiple touchpoints: during prenatal care, in the hospital setting, and at well-child check-ups. Giving parents the chance to understand and discuss infant safe sleep and breastfeeding practices increases the likelihood they will become part of their parenting framework.

NICU nurses play a pivotal role in helping parents transition to home care, and during the time before babies are discharged from the NICU, nurses have a critical opportunity to help parents hear and see by demonstration how to help their baby sleep safely at home. NICU staff are a trusted resource for parents who may not realize what they need to know.

At First Candle, we often hear from parents who have lost their baby to ASSB that they did not know about the dangers of having a blanket or stuffed animal in the crib or having their baby in bed with them. This is why we are increasing our efforts to educate all care providers on the importance of creating a safe sleep environment and why we value the role that neonatology health care providers can play in parent education and baby safety.

\section{"At First Candle, we often hear from parents who have lost their baby to ASSB that they did not know about the dangers of having a blanket or stuffed animal in the crib or having their baby in bed with them."}

References:

1. Erck Lambert AB, Parks SE, Shapiro-Mendoza CK. National and State Trends in Sudden Unexpected Infant Death: 1990-2015. Pediatrics. 2018 Mar;141(3):e20173519. doi: 10.1542/peds.2017-3519. Epub 2018 Feb 12. PMID: 29440504; PMCID: PMC6637428.

2. Erck Lambert AB, Parks SE, Cottengim C, Faulkner M, Hauck F, Shapiro-Mendoza CK. Sleep-Related Infant Suffocation Deaths Attributable to Soft Bedding, Overlay, and Wedging: Pediatrics May 2019, 143 (5) e20183408; DOI: https://doi.org/10.1542/ peds. 2018-3408

3 Goodstein MH, Stewart DL, Keels EL, Moon RY. Transition to a Safe Home Sleep Environment for the NICU Patient: Pediatrics July 2021, 148 (1) e2021052045; DOI: https://doi.org/10.1542/peds.2021-052045.

4. https://www.jognn.org/article/S0884-2175(20)30080-0/fulltext

Disclosure: The author is an International Board Certified Lacta- 
tion Consultant, Certified Doula, and the Director of Education and Bereavement Services of First Candle, Inc., a Connecticut-based not for profit 501(c)3 corporation.

NT

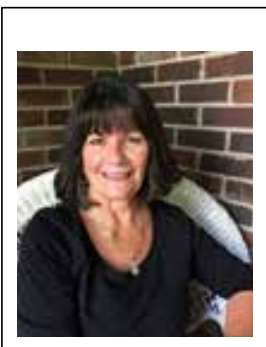

Corresponding Author

Barb Himes, IBCLC

Director of Education and Bereavement Services

First Candle

49 Locust Avenue, Suite 104

New Canaan CT 06840

Telephone: 1-203-966-1300

For Grief Support: 1-800-221-7437

Email: barb@firstcandle.org

www. firstcandle.org

\section{About First Candle}

First Candle, based in New Canaan, CT, is a 501c (3) committed to eliminating Sudden Infant Death Syndrome and other sleeprelated infant deaths while providing bereavement support for families who have suffered a loss. Sudden unexpected infant death (SUID), which includes SIDS and accidental suffocation and strangulation in bed (ASSB), remains the leading cause of death for babies one month to one year of age, resulting in 3,600 infant deaths nationwide per year.

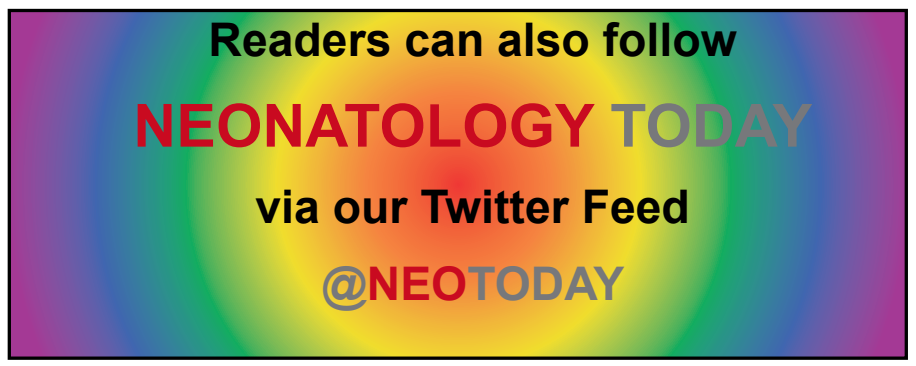

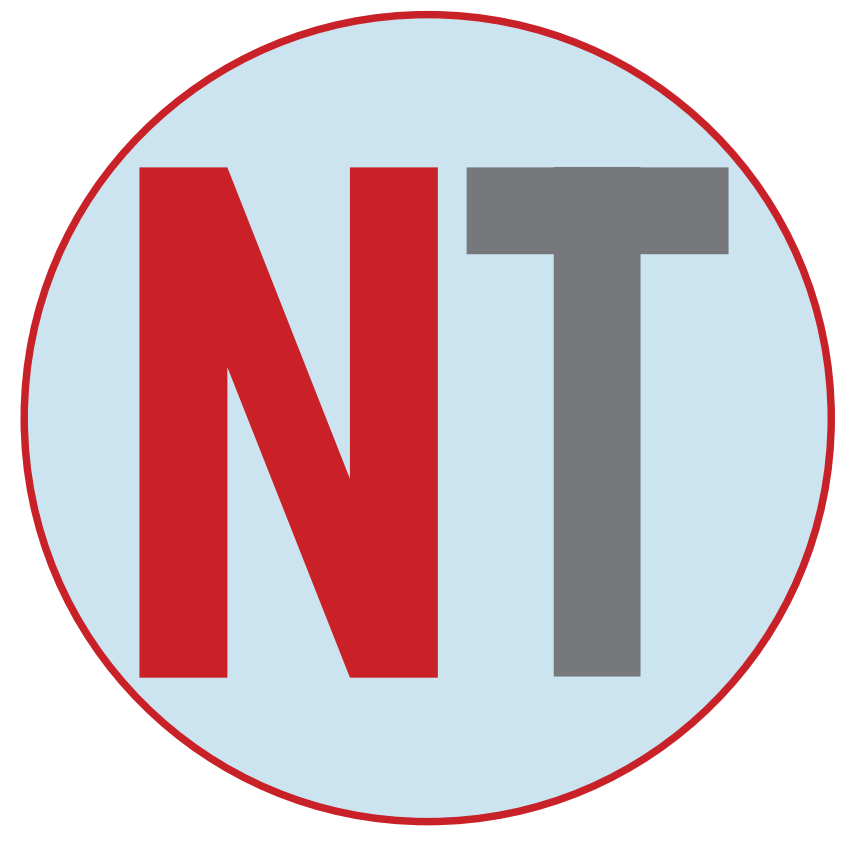

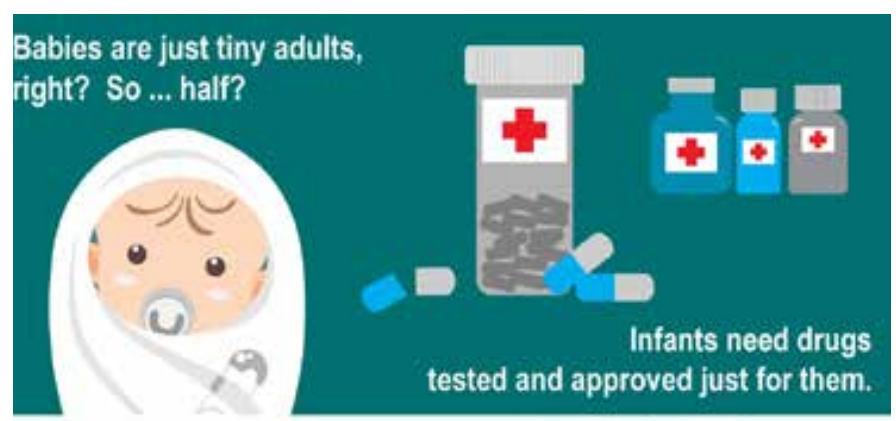

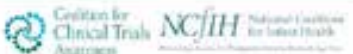

New subscribers are always welcome! NEONATOLOGY IQDAY

To sign up for a free monthly subscription, just click on this box to go directly to our subscription page
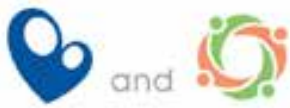

National Perinatal Association and NICU Parent Netwark

mynicunetwork.org 